Plant Tissue Cult. \& Biotech. 31(1): 13-23, 2021 (June)

(C)Bangladesh Assoc. for Plant Tissue Culture \& Biotechnology

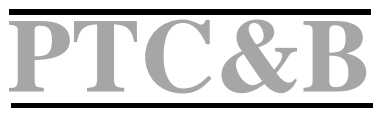

\title{
Micropropagation of Citrus indica Tanaka using Shoot tips from Mature Trees
}

\author{
Kiran Chhetri, Binu Mathew* and Lolly S. Pereira \\ Department of RDAP, North-Eastern Hill University, Tura Campus, Tura, West Garo Hills, \\ Meghalaya, 794002, India.
}

Key words: Citrus indica, shoot tips, in vitro propagation.

\begin{abstract}
A study was conducted to standardize a protocol for in-vitro direct regeneration and mass multiplication of Citrus indica Tanaka using shoot tip explants excised from mature trees. Shoot tips were inoculated in MS medium supplemented with varying concentrations and combinations of cytokinins and auxins. MS media when fortified with BAP $0.5 \mathrm{mg} /$ and $0.5 \mathrm{mg} / \mathrm{BAP}+1.0 \mathrm{mg} / \mathrm{Kn}$ were found to be the best treatments for shoot initiation while MS supplemented with $1 \mathrm{mg} A \mathrm{IBA} ; 0.5 \mathrm{mg} \Lambda \mathrm{NAA}+0.5 \mathrm{mg} / \mathrm{IBA}$ and 0.5 $\mathrm{mg} / \mathrm{NAA}+0.5 \mathrm{mg} /$ IAA were the best treatments for root induction. Among the different media used for hardening, $100 \%$ survivability was obtained when plantlets were hardened using vermicompost as the potting medium. Subsequently, these plantlets were transferred to larger pots and acclimatization was achieved gradually in outdoor conditions.
\end{abstract}

\section{Introduction}

Citrus indica, commonly known as "Indian Wild Orange" and locally called "Memang Narang" is an endemic and endangered species growing in the Nokrek Biosphere Reserve in the North-Eastern Region of India. Various surveys conducted by Singh and Singh (2003) and Upadhyay et al. (2016) indicated that occurrence of this species is restricted to Tura range of Garo hills of Meghalaya, India. The fruits are used for medicinal purposes by the locals to cure diseases like jaundice, kidney stone, small pox and stomach diseases of humans as well as domestic animals (Upadhyay et al. 2016) and in traditional rituals (Malik et al. 2006). Since it is a non- commercial fruit, it has very little economic value. The low genetic diversity of this plant species and increasing human intervention by clearing of forest area for jhum cultivation and human habitation pose a serious threat for

*Author for correspondence: <drbmathew@gmail.com>.

DOI: https://doi.org/10.3329/ptcb.v31i1.54107 
its survival (Malik et al. 2006). Considering the endemic nature and high degree of threat perception, as well as its dwindling population in the natural habitat, this citrus species deserves adequate attention for conservation. In situ conservation of Citrus indica through conventional methods has several limitations such as slower growth rate, short-lived seeds, seed dormancy, seed-borne diseases and high input cost and labour. Therefore, $e x-$ situ conservation may be the most viable alternative for its conservation. In vitro propagation being a non-destructive propagation method, may be considered for mass multiplication of plantlets and planted in various congenial locations for its regeneration and conservation. Mass multiplication of such endangered species is possible only by in vitro micropropagation. However, reports on suitable protocol for mass multiplication of C. indica is very limited.

In a study conducted by Sangma et al. (2020) shoot tip collected from in vitro germinated seedlings proved to be the best explant for in vitro multiplication of $C$. indica. However, in that method availability of sufficient explants for mass multiplication greatly depended on availability of sufficient seeds, which is very limited. Besides that, seedlings do not produce true-to-type progeny. Hence, development of an alternative method ensuring true-to-type progeny is inevitable to ensure mass multiplication of specific and elite type of species. Shoot tips excised from mature trees available in the gene bank shall ensure production of true-to-type plantlets all year round. Therefore, an experiment was laid out with an objective to develop a protocol for in vitro micropropagation of $C$. indica using shoot tip as an explant collected exclusively from mature trees.

\section{Materials and Methods}

The present study was carried out in the Plant Biotechnology Laboratory, Department of Rural Development and Agricultural Production, North-Eastern Hill University, Tura Campus, Chasingre, West Garo Hills District, Meghalaya, India.

Shoot tips of about $1.5-2 \mathrm{~cm}$ in length were excised from mature and healthy trees of Citrus indica maintained at the Department's institutional farm using sterile blades. The shoot tips were immersed in distilled water and brought to the tissue culture laboratory. The explants were then washed in running water thoroughly by adding a few drops of Tween-20 in order to remove the dust particles as well as fungal and bacterial spores present on the surface. This was followed by rinsing with distilled water and moved to the Laminar Air Flow (LAF) cabinet. Under LAF, the explants were surface sterilized by immersing in $0.1 \%(\mathrm{w} / \mathrm{N})$ mercury (II) chloride, $\left(\mathrm{HgCl}_{2}\right)$ for two minutes and then rinsed with sterilized distilled water three times to remove all remains of sterilant solution. Readymade MS fortified with 3\% sucrose and essential vitamins were used for inoculating the explants on different concentrations of growth hormones. The $\mathrm{pH}$ of the medium was adjusted to 5.8 with $1 \mathrm{~N} \mathrm{HCl}$ and $1 \mathrm{~N} \mathrm{NaOH}$ and the final volume of the medium was adjusted to $1 \mathrm{~L}$ using double distilled water. MS basal medium was 
solidified using $1 \%$ agar-agar supplemented with various combinations and concentrations of growth hormones like BAP, Kn, 2,4-D, NAA, IAA and IBA. The medium was then dispensed into sterilized culture vessels and the mouth of the vessels were plugged with non-absorbent cotton plugs. The aseptic manipulations were carried out in the LAF chamber fitted with Ultra Violet light and HEPA (High Efficiency Particulate Air) filter. All the culture vessels were kept in culture room at a temperature of $25 \pm 2^{\circ} \mathrm{C}$, with $60 \pm 5$ percent relative humidity under $35 \mu \mathrm{mol} / \mathrm{ms}$ photosynthetic photon flux white fluorescent light emitted by $40 \mathrm{~W}$ tubes, programmed for 16hour photoperiod and 8hour dark condition.

The shoot tips of Citrus indica were inoculated and multiplied on MS medium (1\% agar-agar) supplemented with varying concentrations (0.5, 1 and $1.5 \mathrm{mg} \Lambda$ ) and combinations (BAP and Kinetin) of growth hormones. The sub-culturing of multiplied shoots was done at an interval of four weeks. The excised micro-shoots were inoculated on MS medium fortified with either cytokinins only or in combination with auxins (2,4-D and NAA) of various concentrations $(0.5-1.5 \mathrm{mg} /)$ and combinations for further multiplication. The in vitro raised multiple shoots of 2 to $3 \mathrm{~cm}$ in length were transferred to MS medium supplemented with different concentrations (0.5-1 mg $\Lambda$ ) of auxins (NAA, IBA and IAA) alone as well as along with cytokinins (BAP and $\mathrm{Kn}$ ) for root induction. The cultures were incubated at $25 \pm 2^{\circ} \mathrm{C}$ under 16-hour photoperiod. Observations were recorded after four weeks of incubation. .

After eight weeks of inoculation in rooting media, the plantlets were 6-8 cm long with well-developed roots which were ready for hardening and transplantation into the soil. The in-vitro regenerated plantlets were removed from the flasks, freed from the adhering medium and transferred into plastic cups filled with only sand, only vermicompost, sand + vermicompost $(1: 1)$; sand + vermicompost + soil mixture (1:1:1) and kept in the growth chamber for fifteen days. Plantlets were subsequently transferred to larger pots and gradually acclimatized to outdoor conditions.

The experiment was laid out in completely randomized design with 34 and 36 treatments each for shoot induction and root induction respectively and the experiment was repeated thrice.

Variance analysis of data was carried out using SPSS (Statistical Package for Social Sciences, SPSS Inc., Chicago, IL, USA) program for statistical analysis. The differences among means for all treatments were tested at $p<0.01 \%$ level of significance and the means were compared by Least Significant Difference (Landau and Everitt 2003). Observations on number of days for shoot and root initiation; number of shoots and roots; length of shoots and roots were recorded. The mean data of shoot and root initiation along with the standard error were presented in the tables. 


\section{Results and Discussion}

Varying concentrations (0.5-1.5 $\mathrm{mg} \Lambda$ ) and combinations of BAP and $\mathrm{Kn}$ were supplemented in MS media for shoot induction. A significant $(\mathrm{p}<0.01)$ variation was observed among the different treatments evaluated for shoot induction. Data presented in Table 1 reveals that the earliest time required for initiation of shoots (13.11 days) was observed on MS media fortified with $0.5 \mathrm{mg} / \mathrm{BAP}+1 \mathrm{mg} / \mathrm{Kn}\left(\mathrm{T}_{9}\right)$. Similar results were seen when $0.5 \mathrm{mg} / \mathrm{BAP}\left(\mathrm{T}_{2}\right)$ and $1.5 \mathrm{mg} / \mathrm{BAP}\left(\mathrm{T}_{4}\right)$ were used without any combination as well but required longer time or produced comparatively low number of shoots. The highest number of shoots (4 nos) were obtained on MS media fortified with $1.5 \mathrm{mg} / \mathrm{BAP}$ $\left(\mathrm{T}_{4}\right)$ and also in $0.5 \mathrm{mg} / \mathrm{BAP}+1 \mathrm{mg} / \mathrm{Kn}\left(\mathrm{T}_{9}\right)$ where $\mathrm{T}_{9}$ medium took shorter duration. Micro-shoots having the highest length $(4.23 \mathrm{~cm})$ were recorded with a combination of 1 $\mathrm{mg} / \mathrm{BAP}+1 \mathrm{mg} / \mathrm{Kn}\left(\mathrm{T}_{12}\right)$.

Table 1. Effects of different cytokinins on shoot initiation of Citrus indica Tanaka cultured on MS medium.

\begin{tabular}{llll}
\hline Treatments & $\begin{array}{l}\text { Days for shoot } \\
\text { initiation }\end{array}$ & No. of shoots & $\begin{array}{l}\text { Length of shoots } \\
(\mathrm{cm})\end{array}$ \\
\hline $\mathrm{T}_{1}(\mathrm{MS})$ Control & $17.77 \pm 0.14^{\mathrm{c}}$ & $1 \pm 0.31^{\mathrm{b}}$ & $1.14 \pm 0.06^{\mathrm{f}}$ \\
$\mathrm{T}_{2}(0.5 \mathrm{BAP})$ & $13.56 \pm 0.37^{\mathrm{ab}}$ & $2.44 \pm 0.17^{\mathrm{ab}}$ & $3.23 \pm 0.09^{\mathrm{ab}}$ \\
$\mathrm{T}_{3}(1 \mathrm{BAP})$ & $15.11 \pm 0.35^{\mathrm{abc}}$ & $3.33 \pm 0.16^{\mathrm{ab}}$ & $3.34 \pm 0.11^{\mathrm{abcd}}$ \\
$\mathrm{T}_{4}(1.5 \mathrm{BAP})$ & $14.88 \pm 0.38^{\mathrm{abc}}$ & $4 \pm 0.28^{\mathrm{a}}$ & $2.12 \pm 0.33^{\mathrm{e}}$ \\
$\mathrm{T}_{5}(0.5 \mathrm{Kn})$ & $16.44 \pm 0.47^{\mathrm{abc}}$ & $2.55 \pm 0.17^{\mathrm{ab}}$ & $2.47 \pm 0.20^{\mathrm{de}}$ \\
$\mathrm{T}_{6}(1 \mathrm{Kn})$ & $16.37 \pm 0.82^{\mathrm{abc}}$ & $1.37 \pm 0.17^{\mathrm{b}}$ & $2.93 \pm 0.14^{\mathrm{cde}}$ \\
$\mathrm{T}_{7}(1.5 \mathrm{Kn})$ & $16.11 \pm 0.36^{\mathrm{abc}}$ & $2.66 \pm 0.33^{\mathrm{ab}}$ & $3.20 \pm 0.09^{\mathrm{bcd}}$ \\
$\mathrm{T}_{8}(0.5 \mathrm{BAP}+0.5 \mathrm{Kn})$ & $15.33 \pm 0.16^{\mathrm{abc}}$ & $2.66 \pm 0.44^{\mathrm{ab}}$ & $3.30 \pm 0.15^{\mathrm{bcd}}$ \\
$\mathrm{T}_{9}(0.5 \mathrm{BAP}+1 \mathrm{Kn})$ & $13.11 \pm 0.38^{\mathrm{a}}$ & $4 \pm 0.28^{\mathrm{a}}$ & $3.90 \pm 0.05^{\mathrm{ab}}$ \\
$\mathrm{T}_{10}(0.5 \mathrm{BAP}+1.5 \mathrm{Kn})$ & $16.33 \pm 0.16^{\mathrm{abc}}$ & $3 \pm 0.28^{\mathrm{ab}}$ & $3.35 \pm 0.11^{\mathrm{abcd}}$ \\
$\mathrm{T}_{11}(1 \mathrm{BAP}+0.5 \mathrm{Kn})$ & $16.67 \pm 0.33^{\mathrm{bc}}$ & $3 \pm 0.28^{\mathrm{ab}}$ & $3.50 \pm 0.33^{\mathrm{abcd}}$ \\
$\mathrm{T}_{12}(1 \mathrm{BAP}+1 \mathrm{Kn})$ & $15.44 \pm 0.47^{\mathrm{abc}}$ & $3.33 \pm 0.44^{\mathrm{ab}}$ & $4.23 \pm 0.04^{\mathrm{a}}$ \\
$\mathrm{T}_{13}(1 \mathrm{BAP}+1.5 \mathrm{Kn})$ & $15.33 \pm 0.16^{\mathrm{abc}}$ & $3 \pm 0.28^{\mathrm{ab}}$ & $3.92 \pm 0.05^{\mathrm{ab}}$ \\
$\mathrm{T}_{14}(1.5 \mathrm{BAP}+0.5 \mathrm{Kn})$ & $15 \pm 0.5^{\mathrm{abc}}$ & $3 \pm 0.27^{\mathrm{ab}}$ & $3.70 \pm 0.06^{\mathrm{abcd}}$ \\
$\mathrm{T}_{15}(1.5 \mathrm{BAP}+1 \mathrm{Kn})$ & $15 \pm 0.42^{\mathrm{abc}}$ & $2.77 \pm 0.27^{\mathrm{ab}}$ & $3.30 \pm 0.09^{\mathrm{bcd}}$ \\
$\mathrm{T}_{16}(1.5 \mathrm{BAP}+1.5 \mathrm{Kn})$ & $15.33 \pm 0.86^{\mathrm{abc}}$ & $3.22 \pm 0.38^{\mathrm{ab}}$ & $3.02 \pm 0.14^{\mathrm{bcde}}$ \\
\hline
\end{tabular}

Values represent mean \pm standard error. Columns with similar letters are not significantly different.

Data in Table 2 showed that the earliest shoot induction (14.44 days) was recorded in $\mathrm{T}_{4}$ when MS media was supplemented with $0.5 \mathrm{mg} / \mathrm{Kn}+1 \mathrm{mg} / \mathrm{BAP}+0.5 \mathrm{mg} / 2,4-\mathrm{D}$ followed by $\mathrm{T}_{16}$ (15 days) where MS media was supplemented with $0.5 \mathrm{mg} / \mathrm{Kn}+1.5 \mathrm{mg} /$ $\mathrm{BAP}+0.5 \mathrm{mg} / \mathrm{NAA}$. Similar results were reported in $\mathrm{T}_{1}(0.5 \mathrm{mg} / \mathrm{Kn}+0.5 \mathrm{mg} / \mathrm{BAP}+0.5$ $\mathrm{mg} / 2,4-\mathrm{D}), \mathrm{T}_{3}(0.5 \mathrm{mg} / \mathrm{Kn}+0.5 \mathrm{mg} / \mathrm{BAP}+1.5 \mathrm{mg} / 2,4-\mathrm{D})$ and $\mathrm{T}_{12}(0.5 \mathrm{mg} / \mathrm{Kn}+0.5$ $\mathrm{mg} / \mathrm{BAP}+1.5 \mathrm{mg} / \mathrm{NAA})$. MS media supplemented with $0.5 \mathrm{mg} / \mathrm{Kn}+1 \mathrm{mg} / \mathrm{BAP}+1$ $\mathrm{mg} / 2,4-\mathrm{D}\left(\mathrm{T}_{5}\right)$ gave the highest number of micro-shoots $(4 \mathrm{nos})$ followed by $\mathrm{T}_{6}(0.5 \mathrm{mg} /$ 
Kinetin $+1 \mathrm{mg} / \mathrm{BAP}+1.5 \mathrm{mg} / 2,4-\mathrm{D})$. The number of shoots was statistically at par when the concentration of $\mathrm{Kn}(0.5 \mathrm{mg} \Lambda)$ and BAP $(1.5 \mathrm{mg} \Lambda)$ were kept constant but the concentration of 2,4-D was increased from 0.5 to $1.5 \mathrm{mg} /$. This shows that difference in concentration of 2,4-D between $0.5 \mathrm{mg} \Lambda$ to $1.5 \mathrm{mg} \Lambda$ doesn't have any significant difference. The tallest micro-shoots were observed in $\mathrm{T}_{9}(0.5 \mathrm{mg} / \mathrm{Kn}+1.5 \mathrm{mg} / \mathrm{BAP}+$ $1.5 \mathrm{mg} / 2,4-\mathrm{D})$ and $\mathrm{T}_{16}(0.5 \mathrm{mg} / \mathrm{Kn}+1.5 \mathrm{mg} / \mathrm{BAP}+0.5 \mathrm{mg} / \mathrm{NAA})$. In the present study it was observed that MS fortified with cytokinins $(0.5 \mathrm{mg} / \mathrm{BAP}+1 \mathrm{mg} / \mathrm{Kn})$ gave early response for shoot induction as compared to the combination with auxins.

Table 2. Effects of different combinations of cytokinins and auxins on shoot initiation in Citrus indica Tanak cultured on MS medium.

\begin{tabular}{|c|c|c|c|}
\hline Treatments & $\begin{array}{l}\text { Days for shoot } \\
\text { initiation }\end{array}$ & No. of shoots & $\begin{array}{l}\text { Length of shoots } \\
(\mathrm{cm})\end{array}$ \\
\hline $\mathrm{T}_{1}(0.5 \mathrm{Kn}+0.5 \mathrm{BAP}+0.52,4-\mathrm{D})$ & $15.67 \pm 0.16^{\mathrm{abc}}$ & $2 \pm 0.28^{\mathrm{ab}}$ & $1.56 \pm 0.07 \mathrm{~g}$ \\
\hline $\mathrm{T}_{2}(0.5 \mathrm{Kn}+0.5 \mathrm{BAP}+12,4-\mathrm{D})$ & $17.33 \pm 0.33^{\mathrm{cd}}$ & $2.67 \pm 0.33^{\mathrm{ab}}$ & $2.51 \pm 0.07^{\mathrm{fg}}$ \\
\hline $\mathrm{T}_{3}(0.5 \mathrm{Kn}+0.5 \mathrm{BAP}+1.52,4-\mathrm{D})$ & $15.33 \pm 0.72^{\mathrm{abc}}$ & $2.67 \pm 0.16^{\mathrm{ab}}$ & $3.34 \pm 0.33^{\text {cde }}$ \\
\hline $\mathrm{T}_{4}(0.5 \mathrm{Kn}+1 \mathrm{BAP}+0.52,4-\mathrm{D})$ & $14.44 \pm 0.50^{a}$ & $2.44 \pm 0.17 \mathrm{ab}$ & $3.41 \pm 0.11^{\text {cde }}$ \\
\hline $\mathrm{T}_{5}(0.5 \mathrm{Kn}+1 \mathrm{BAP}+12,4-\mathrm{D})$ & $16 \pm 0.28^{\mathrm{abcd}}$ & $4 \pm 0.28^{a}$ & $3.3 \pm 0.14 c^{\mathrm{de}}$ \\
\hline $\mathrm{T}_{6}(0.5 \mathrm{Kn}+1 \mathrm{BAP}+1.52,4-\mathrm{D})$ & $16 \pm 0.33^{\mathrm{abcd}}$ & $3.38 \pm 0.33^{\mathrm{ab}}$ & $3.42 \pm 0.04^{\text {cde }}$ \\
\hline $\mathrm{T}_{7}(0.5 \mathrm{Kn}+1.5 \mathrm{BAP}+0.5$ 2,4-D) & $16.56 \pm 0.41^{\mathrm{abcd}}$ & $3.33 \pm 0.33^{\mathrm{ab}}$ & $3.21 \pm 0.11^{\mathrm{e}}$ \\
\hline $\mathrm{T}_{8}(0.5 \mathrm{Kn}+1.5 \mathrm{BAP}+12,4-\mathrm{D})$ & $16.56 \pm 0.17^{\mathrm{abcd}}$ & $3.33 \pm 0.26^{\mathrm{ab}}$ & $3.43 \pm 0.05^{\text {bcde }}$ \\
\hline $\mathrm{T}_{9}(0.5 \mathrm{Kn}+1.5 \mathrm{BAP}+1.5$ 2,4-D) & $16.33 \pm 0.44^{\mathrm{abcd}}$ & $3.00 \pm 0.28^{\mathrm{ab}}$ & $4.01 \pm 0.06^{\mathrm{a}}$ \\
\hline $\mathrm{T}_{10}(0.5 \mathrm{Kn}+0.5 \mathrm{BAP}+0.5 \mathrm{NAA})$ & $18 \pm 0.28^{\mathrm{d}}$ & $3 \pm 0.28^{\mathrm{ab}}$ & $3.35 \pm 0.10^{\mathrm{de}}$ \\
\hline $\mathrm{T}_{11}(0.5 \mathrm{Kn}+0.5 \mathrm{BAP}+1 \mathrm{NAA})$ & $17 \pm 0.5^{\mathrm{bcd}}$ & $2.66 \pm 0.16^{\mathrm{ab}}$ & $3.07 \pm 0.10^{\mathrm{ef}}$ \\
\hline $\mathrm{T}_{12}(0.5 \mathrm{Kn}+0.5 \mathrm{BAP}+1.5 \mathrm{NAA})$ & $15.67 \pm 0.16^{\mathrm{abc}}$ & $2.33 \pm 0.16^{\mathrm{ab}}$ & $3.06 \pm 0.07^{\mathrm{ef}}$ \\
\hline $\mathrm{T}_{13}(0.5 \mathrm{Kn}+1 \mathrm{BAP}+0.5 \mathrm{NAA})$ & $16.33 \pm 0.33^{\mathrm{abcd}}$ & $2.27 \pm 0.22^{\mathrm{ab}}$ & $3.01 \pm 0.16^{\mathrm{ef}}$ \\
\hline $\mathrm{T}_{14}(0.5 \mathrm{Kn}+1 \mathrm{BAP}+1 \mathrm{NAA})$ & $17.33 \pm 0.44^{\mathrm{cd}}$ & $2.33 \pm 0.33^{\mathrm{ab}}$ & $3.83 \pm 0.09 \mathrm{abcd}$ \\
\hline $\mathrm{T}_{15}(0.5 \mathrm{Kn}+1 \mathrm{BAP}+1.5 \mathrm{NAA})$ & $17.44 \pm 0.50^{\mathrm{cd}}$ & $3.11 \pm 0.26^{\mathrm{ab}}$ & $3.91 \pm 0.13^{\mathrm{abc}}$ \\
\hline $\mathrm{T}_{16}(0.5 \mathrm{Kn}+1.5 \mathrm{BAP}+0.5 \mathrm{NAA})$ & $15 \pm 0.50^{\mathrm{ab}}$ & $2 \pm 0.28^{\mathrm{ab}}$ & $4.07 \pm 0.05^{\mathrm{a}}$ \\
\hline $\mathrm{T}_{17}(0.5 \mathrm{Kn}+1.5 \mathrm{BAP}+1 \mathrm{NAA})$ & $17 \pm 0.16^{\mathrm{bcd}}$ & $2.67 \pm 0.28^{\mathrm{ab}}$ & $3.40 \pm 0.05^{\text {cde }}$ \\
\hline $\mathrm{T}_{18}(0.5 \mathrm{Kn}+1.5 \mathrm{BAP}+1.5 \mathrm{NAA})$ & $17 \pm 0.16^{\mathrm{bcd}}$ & $3.33 \pm 0.28^{\mathrm{ab}}$ & $3.45 \pm 0.05^{\text {bcde }}$ \\
\hline
\end{tabular}

Values represent mean \pm SE. Columns with similar letters are not significantly different.

The highest number of shoots were obtained in $1.5 \mathrm{mg} / \mathrm{BAP}, 0.5 \mathrm{mg} \Lambda \mathrm{BAP}+1 \mathrm{mg} \Lambda$ $\mathrm{Kn}$ and $0.5 \mathrm{mg} / \mathrm{Kn}+1 \mathrm{mg} / \mathrm{BAP}+1 \mathrm{mg} / 2$,4-D. Similar result was reported in Citrus volkameriana (Schinor et al. 2011) where the number of shoots increased when $1.5 \mathrm{mg} A$ BAP was used. The multiplication of shoots appeared to be affected by the use of BAP. 
The observations bear similarity with Citrus aurantifolia as reported by Al-Khayri et al. (2005). BAP has been reported to play a major role in the shooting of other Citrus species as well like Citrus jambhiri (Saini et al. 2010, Rattanpal et al. 2011), Citrus clementina (Lomabardo et al. 2011) and Citrus hystrix (Eng et al. 2014).

The best treatments for achieving early shoot initiation, highest number of shoots per explant and longest shoots was found to be $0.5 \mathrm{mg} / \mathrm{BAP}$ and $0.5 \mathrm{mg} / \mathrm{BAP}+1.0 \mathrm{mg} / \mathrm{Kn}$. Efficacy of $0.5 \mathrm{mg} \Lambda$ BAP was also reported by Sangma et al. (2020) in a similar work carried out on micropropagation of Citrus indica. Although both the studies exhibited similar trend, the number of days required for shoot initiation and the mean number of shoots per explants varied significantly. This may be due to the difference in source of explants. Explants collected from in vitro seedlings produced multiple shoots at very short time.

Data presented in Table 3 reveal that the earliest response (17.33 days) for root induction was recorded in MS medium supplemented with $1 \mathrm{mg} / \mathrm{NAA}+1 \mathrm{mg} / \mathrm{IAA}$ ( $\left.\mathrm{T}_{13}\right)$. MS media supplemented with $1 \mathrm{mg} / \mathrm{IAA}+0.5 \mathrm{mg} / \mathrm{IBA}\left(\mathrm{T}_{18}\right)$ gave the highest number of roots (5.62 nos). Similar results were obtained when $1 \mathrm{mg} / \mathrm{IAA}$ ( $\mathrm{T}_{5}$ ) and 0.5 $\mathrm{mg} / \mathrm{NAA}+1 \mathrm{mg} / \mathrm{IAA}\left(\mathrm{T}_{9}\right)$ were used but required longer time than $\mathrm{T}_{13}$ medium. The highest root length $(6.16 \mathrm{~cm})$ was recorded in MS media fortified with $0.5 \mathrm{mg} / \mathrm{NAA}+0.5$ $\mathrm{mg} / \mathrm{IBA}\left(\mathrm{T}_{10}\right)$ followed by $0.5 \mathrm{mg} / \mathrm{NAA}+0.5 \mathrm{mg} / \mathrm{IAA}$ ( $\left.\mathrm{T}_{8}\right)$ and $1 \mathrm{mg} / \mathrm{IBA}\left(\mathrm{T}_{7}\right)$.

Table 4 indicates that the earliest initiation of roots (18.44 days) was observed in $T_{9}$ $(0.5 \mathrm{mg} / \mathrm{Kn}+1.5 \mathrm{mg} / \mathrm{BAP}+1.5 \mathrm{mg} / \mathrm{IAA})$ followed by $\mathrm{T}_{1}(0.5 \mathrm{mg} / \mathrm{Kn}+0.5 \mathrm{mg} / \mathrm{BAP}+$ $0.5 \mathrm{mg} / \mathrm{IAA})$ and $\mathrm{T}_{14}(0.5 \mathrm{mg} /$ Kinetin $+1 \mathrm{mg} / \mathrm{BAP}+1 \mathrm{mg} / \mathrm{NAA})$. The highest number of roots (6.67) were recorded in MS medium fortified with $0.5 \mathrm{mg} \Lambda$ Kinetin $+1.5 \mathrm{mg} \Lambda$ $\mathrm{BAP}+1 \mathrm{mg} / \mathrm{IAA}\left(\mathrm{T}_{8}\right)$ followed by $\mathrm{T}_{12}(0.5 \mathrm{mg} / \mathrm{Kn}+0.5 \mathrm{mg} / \mathrm{BAP}+1.5 \mathrm{mg} / \mathrm{NAA})$ and $\mathrm{T}_{14}(0.5 \mathrm{mg} / \mathrm{Kn}+1 \mathrm{mg} / \mathrm{BAP}+1 \mathrm{mg} / \mathrm{NAA})$. The highest root length was recorded in $\mathrm{T}_{6}$ $(0.5 \mathrm{mg} / \mathrm{Kn}+1 \mathrm{mg} / \mathrm{BAP}+1.5 \mathrm{mg} / \mathrm{IAA})$ followed by $\mathrm{T}_{17}(0.5 \mathrm{mg} / \mathrm{Kn}+1.5 \mathrm{mg} / \mathrm{BAP}+$ $1 \mathrm{mg} / \mathrm{NAA})$. Similar results were seen when $0.5 \mathrm{mg} / \mathrm{Kn}+1 \mathrm{mg} / \mathrm{BAP}+1.5 \mathrm{mg} / \mathrm{NAA}$ ( $\mathrm{T}_{15}$ ) were used. Fig. 1 represents different stages of micropropagation of Citrus indica.

The earliest root induction was observed in MS medium supplemented with $1 \mathrm{mg} /$ $\mathrm{NAA}+1 \mathrm{mg} /$ IAA and $1 \mathrm{mg} / \mathrm{NAA}+0.5 \mathrm{mg} /$ IBA. Similar observations were reported in Citrus jambhiri Lush (Kaur 2016). IBA has been observed to be an early root inducer in Citrus limon L. cv. Kaghzi Kalan (Goswami et al. 2013) as well. When auxins were used alone, the highest number of roots were achieved in $1 \mathrm{mg} / \mathrm{IAA}+0.5 \mathrm{mg} / \mathrm{IBA}$. The combination of IAA and IBA produced highest number of roots in Citrus limon (PerezTornero et al. 2009, Kaur 2016) and Citrus macrophylla (Tallon et al. 2009). Early root initiation and higher number of roots were achieved when $1 \mathrm{mg} /$ NAA was used in MS media. Similar observation was also reported by Sangma et al. (2020), in Citrus indica. The combination of cytokinins did not show any significant improvement in root initiation. However, higher number of roots were obtained when both NAA and IAA were used in combination with cytokinins. The longest roots were observed in MS media supplemented with $0.5 \mathrm{mg} / \mathrm{NAA}+0.5 \mathrm{mg} \Lambda$ IBA when auxins alone were used. 
Table 3. Effect of different auxins on root initiation of in vitro raised shoots of Citrus indica cultured in MS medium.

\begin{tabular}{|c|c|c|c|}
\hline Treatments & Days for root initiation & No. of roots & Root length \\
\hline $\mathrm{T}_{1}(\mathrm{MS})$ Control & $20.33 \pm 0.6^{\mathrm{abc}}$ & $1.67 \pm 0.33 c$ & $0.21 \pm 0.13 g$ \\
\hline $\mathrm{T}_{2}(0.5 \mathrm{NAA})$ & $21.66 \pm 0.33^{c}$ & $3.67 \pm 0.16^{\mathrm{abc}}$ & $3.42 \pm 0.13^{\mathrm{fg}}$ \\
\hline $\mathrm{T}_{3}(1 \mathrm{NAA})$ & $20.77 \pm 0.27 \mathrm{abc}$ & $4.67 \pm 0.33^{\mathrm{ab}}$ & $3.9 \pm 0.05^{\operatorname{defg}}$ \\
\hline $\mathrm{T}_{4}(0.5 \mathrm{IAA})$ & $20.33 \pm 0.33^{\mathrm{abc}}$ & $3 \pm 0.28^{\mathrm{bc}}$ & $3.23 \pm 0.18^{f}$ \\
\hline $\mathrm{T}_{5}(1 \mathrm{IAA})$ & $20 \pm 0.28^{\mathrm{abc}}$ & $5 \pm 0.40^{\mathrm{ab}}$ & $3.65 \pm 0.1^{\mathrm{efg}}$ \\
\hline $\mathrm{T}_{6}(0.5 \mathrm{IBA})$ & $21.5 \pm 0.43^{\mathrm{bc}}$ & $4.6 \pm 0.46^{\mathrm{ab}}$ & $4.46 \pm 0.15^{\text {cde }}$ \\
\hline $\mathrm{T}_{7}(1 \mathrm{IBA})$ & $19.33 \pm 0.72^{\mathrm{abc}}$ & $4.33 \pm 0.44^{\mathrm{ab}}$ & $5.57 \pm 0.19 \mathrm{ab}$ \\
\hline $\mathrm{T}_{8}(0.5 \mathrm{NAA}+0.5 \mathrm{IAA})$ & $19.33 \pm 0.60^{\mathrm{abc}}$ & $4.67 \pm 0.16^{\mathrm{ab}}$ & $5.64 \pm 0.05^{\mathrm{ab}}$ \\
\hline $\mathrm{T}_{9}(0.5 \mathrm{NAA}+1 \mathrm{IAA})$ & $19.67 \pm 0.33^{\mathrm{abc}}$ & $5.44 \pm 0.33^{a}$ & $3.84 \pm 0.09 \operatorname{defg}$ \\
\hline $\mathrm{T}_{10}(0.5 \mathrm{NAA}+0.5 \mathrm{IBA})$ & $18.22 \pm 0.27 \mathrm{abc}$ & $4.66 \pm 0.33^{\mathrm{ab}}$ & $6.16 \pm 0.1^{\mathrm{a}}$ \\
\hline $\mathrm{T}_{11}(0.5 \mathrm{NAA}+1 \mathrm{IBA})$ & $18.55 \pm 0.17 \mathrm{abc}$ & $3.33 \pm 0.16^{\mathrm{abc}}$ & $4.86 \pm 0.11^{\mathrm{bc}}$ \\
\hline $\mathrm{T}_{12}(1 \mathrm{NAA}+0.5 \mathrm{IAA})$ & $19.55 \pm 0.55^{\mathrm{abc}}$ & $3.66 \pm 0.33^{\mathrm{abc}}$ & $3.81 \pm 0.12^{\mathrm{defg}}$ \\
\hline $\mathrm{T}_{13}(1 \mathrm{NAA}+1 \mathrm{IAA})$ & $17.33 \pm 0.44^{\mathrm{a}}$ & $4.33 \pm 0.33^{\mathrm{ab}}$ & $4.75 \pm 0.12^{\mathrm{bcd}}$ \\
\hline $\mathrm{T}_{14}(1 \mathrm{NAA}+0.5 \mathrm{IBA})$ & $17.66 \pm 0.16^{\mathrm{ab}}$ & $3.44 \pm 0.16^{\mathrm{abc}}$ & $3.91 \pm 0.07^{\text {cdeffg }}$ \\
\hline $\mathrm{T}_{15}(1 \mathrm{NAA}+1 \mathrm{IBA})$ & $19 \pm 0.57 \mathrm{abc}$ & $3.55 \pm 0.17 \mathrm{abc}$ & $4.44 \pm 0.07^{\text {cde }}$ \\
\hline $\mathrm{T}_{16}(0.5 \mathrm{IAA}+0.5 \mathrm{IBA})$ & $19 \pm 0.57 \mathrm{abc}$ & $2.67 \pm 0.17 \mathrm{bc}$ & $4.23 \pm 0.94^{\text {cdef }}$ \\
\hline $\mathrm{T}_{17}(1 \mathrm{IAA}+1 \mathrm{IBA})$ & $20.22 \pm 0.46^{\mathrm{abc}}$ & $4 \pm 0.33^{\mathrm{abc}}$ & $3.98 \pm 0.07_{\text {cdefg }}$ \\
\hline $\mathrm{T}_{18}(1 \mathrm{IAA}+0.5 \mathrm{IBA})$ & $18.25 \pm 0.34^{\mathrm{abc}}$ & $5.62 \pm 0.17^{a}$ & $3.85 \pm 0.01^{\operatorname{defg}}$ \\
\hline
\end{tabular}

Values represent mean \pm standard error. Columns with similar letters are not significantly different.

Out of the four different treatments used for hardening viz., $\mathrm{T}_{1}$ (sand), $\mathrm{T}_{2}$ (vermicompost), $\mathrm{T}_{3}$ \{sand+vermicompost $\left.(1: 1)\right\}, \mathrm{T}_{4}$ \{sand+vermicompost+soil $\left.(1: 1: 1)\right\}$, $100 \%$ survivability was obtained in plantlets that were hardened in vermicompost alone when observed after eight weeks (Fig. 2). Plants grown in vermicompost + sand (1:1) exhibited $94.4 \%$ survivability. Subsequently, these plantlets were transferred to larger pots after 4 months and acclimatization was achieved gradually in outdoor conditions where the rate of survival of the plantlets was $100 \%$. Kangabam et al. (2020) reported 92.89\% survivability in Citrus jambhiri Lush in vitro regenerated plantlets when vermicompost and vermiculite were used as a hardening mixture in the ratio of 1:1. Atiyeh et al. (2002) and Musculo et al. (1996) reported that high level of humic substances present in the vermicompost derived from different organic wastes showed growth hormone like properties thereby enhancing the growth of plantlets. 

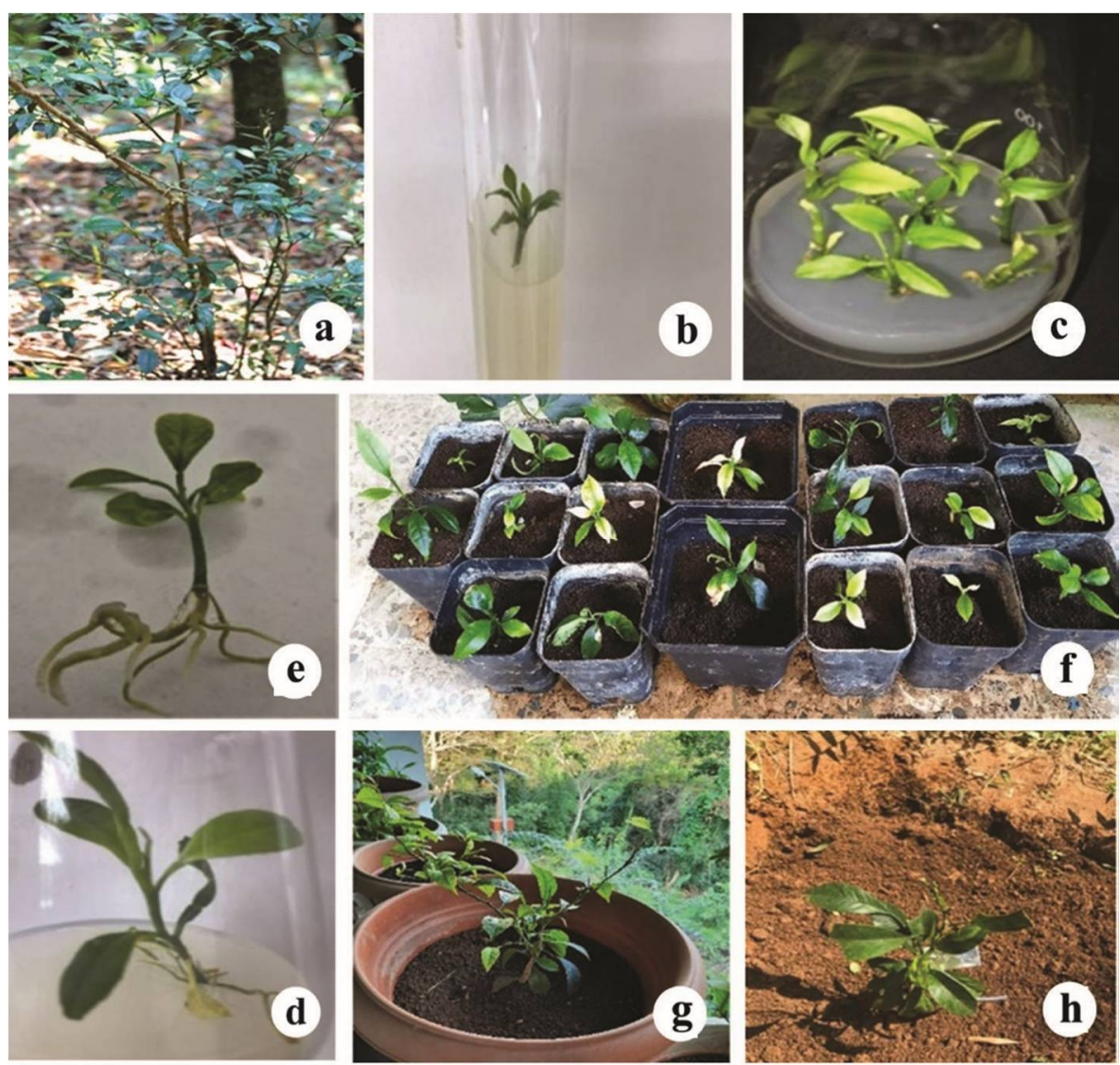

Fig.1a-h: Presents the different stages of micropropagation (a) Extraction of shoot tips from the mature Citrus indica tree. (b) To initial shoot regeneration. (c) Development of micro-shoots. (d) Induction of root from in vitro regenerated micro-shoot. (e) Fully rooted pant lets. (f,g) To different stages of hardening and (h) Transplantation of regenerated Citrus indica plantlets in the main field.

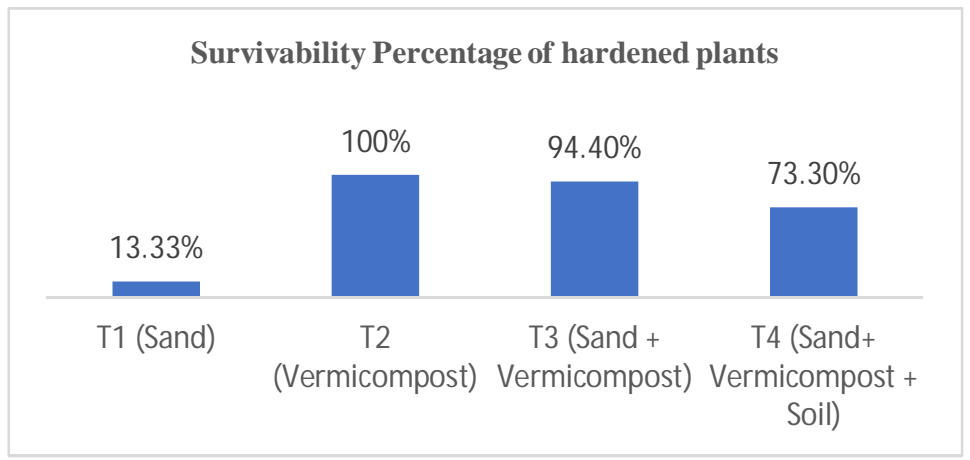

Fig. 2. Effect of different potting media on the survival of the hardened plants. 
Table 4. Effect of different concentrations and combinations of cytokinins and auxins in root initiation of in vitro raised shoots of Citrus indica cultured in MS medium.

\begin{tabular}{|c|c|c|c|}
\hline Treatments & Days for root initiation & No. of roots & Root length \\
\hline $\mathrm{T} 1(0.5 \mathrm{Kn}+0.5 \mathrm{BAP}+0.5 \mathrm{IAA})$ & $19.67 \pm 0.33^{\mathrm{abc}}$ & $3 \pm 0.28^{\text {gh }}$ & $4 \pm 0.06^{\text {def }}$ \\
\hline $\mathrm{T}_{2}(0.5 \mathrm{Kn}+0.5 \mathrm{BAP}+1 \mathrm{IAA})$ & $21 \pm 0.28^{\text {bcde }}$ & $3.66 \pm 0.16^{\text {efgh }}$ & $3.78 \pm 0.11^{\text {defg }}$ \\
\hline $\mathrm{T}_{3}(0.5 \mathrm{Kn}+0.5 \mathrm{BAP}+1.5 \mathrm{IAA})$ & $20.66 \pm 0.16^{\text {bcde }}$ & $4.66 \pm 0.16^{\text {cdefgh }}$ & $3.61 \pm 0.26^{\mathrm{efg}}$ \\
\hline $\mathrm{T}_{4}(0.5 \mathrm{Kn}+1 \mathrm{BAP}+0.5 \mathrm{IAA})$ & $20.11 \pm 0.23^{\mathrm{abcd}}$ & $5.33 \pm 0.33^{\text {abcde }}$ & $4.23 \pm 0.13^{\mathrm{cd}}$ \\
\hline $\mathrm{T}_{5}(0.5 \mathrm{Kn}+1 \mathrm{BAP}+1 \mathrm{IAA})$ & $20.77 \pm 0.49^{\text {bcde }}$ & $5.66 \pm 0.16^{\mathrm{abcd}}$ & $5.13 \pm 0.08^{\mathrm{b}}$ \\
\hline $\mathrm{T}_{6}(0.5 \mathrm{Kn}+1 \mathrm{BAP}+1.5 \mathrm{IAA})$ & $19.22 \pm 0.22^{\mathrm{ab}}$ & $2.77 \pm 0.14^{\mathrm{h}}$ & $5.91 \pm 0.07^{\mathrm{a}}$ \\
\hline $\mathrm{T}_{7}(0.5 \mathrm{Kn}+1.5 \mathrm{BAP}+0.5 \mathrm{IAA})$ & $21 \pm 0.28^{\text {bcde }}$ & $3.33 \pm 0.16^{\text {fgh }}$ & $4.12 \pm 0.10^{\text {de }}$ \\
\hline $\mathrm{T}_{8}(0.5 \mathrm{Kn}+1.5 \mathrm{BAP}+1 \mathrm{IAA})$ & $21.33 \pm 0.16^{\text {cde }}$ & $6.67 \pm 0.16^{\mathrm{a}}$ & $3.91 \pm 0.03^{\text {def }}$ \\
\hline $\mathrm{T}_{9}(0.5 \mathrm{Kn}+1.5 \mathrm{BAP}+1.5 \mathrm{IAA})$ & $18.44 \pm 0.17^{a}$ & $5.44 \pm 0.17 \mathrm{abcd}$ & $3.83 \pm 0.01^{\text {def }}$ \\
\hline $\mathrm{T}_{10}(0.5 \mathrm{Kn}+0.5 \mathrm{BAP}+0.5 \mathrm{NAA})$ & $20.78 \pm 0.52^{\text {bcde }}$ & $3.33 \pm 0.16^{\mathrm{fgh}}$ & $3.27 \pm 0.11^{g}$ \\
\hline $\mathrm{T}_{11}(0.5 \mathrm{Kn}+0.5 \mathrm{BAP}+1 \mathrm{NAA})$ & $21 \pm 0.28^{\text {bde }}$ & $4 \pm 0.23^{\text {defgh }}$ & $3.51 \pm 0.04^{\mathrm{fg}}$ \\
\hline $\mathrm{T}_{12}(0.5 \mathrm{Kn}+0.5 \mathrm{BAP}+1.5 \mathrm{NAA})$ & $22.33 \pm 0.16^{\mathrm{e}}$ & $6 \pm 0.40^{\mathrm{abc}}$ & $3.96 \pm 0.11^{\text {def }}$ \\
\hline $\mathrm{T}_{13}(0.5 \mathrm{Kn}+1 \mathrm{BAP}+0.5 \mathrm{NAA})$ & $21.77 \pm 0.4^{\mathrm{de}}$ & $4.77 \pm 0.43^{\mathrm{bcdef}}$ & $4.92 \pm 0.06^{\mathrm{b}}$ \\
\hline $\mathrm{T}_{14}(0.5 \mathrm{Kn}+1 \mathrm{BAP}+1 \mathrm{NAA})$ & $19.67 \pm 0.16^{\mathrm{abc}}$ & $6.44 \pm 0.41^{\mathrm{ab}}$ & $4.72 \pm 0.04^{\mathrm{bc}}$ \\
\hline $\mathrm{T}_{15}(0.5 \mathrm{Kn}+1 \mathrm{BAP}+1.5 \mathrm{NAA})$ & $22.22 \pm 0.32^{\mathrm{e}}$ & $4.66 \pm 0.44^{\text {cdefg }}$ & $5.76 \pm 0.07^{a}$ \\
\hline $\mathrm{T}_{16}(0.5 \mathrm{Kn}+1.5 \mathrm{BAP}+0.5 \mathrm{NAA})$ & $21.33 \pm 0.16^{\text {cde }}$ & $5.66 \pm 0.44^{\mathrm{abcd}}$ & $5.16 \pm 0.15^{\mathrm{b}}$ \\
\hline $\mathrm{T}_{17}(0.5 \mathrm{Kn}+1.5 \mathrm{BAP}+1 \mathrm{NAA})$ & $21.66 \pm 0.44^{\mathrm{de}}$ & $5.22 \pm 0.22^{\mathrm{abcde}}$ & $5.88 \pm 0.08^{a}$ \\
\hline $\mathrm{T}_{18}(0.5 \mathrm{Kn}+1.5 \mathrm{BAP}+1.5 \mathrm{NAA})$ & $20.88 \pm 0.48^{\text {bcde }}$ & $5.33 \pm 0.33^{\text {abcde }}$ & $5.17 \pm 0.1^{\mathrm{b}}$ \\
\hline
\end{tabular}

Values represent mean \pm SE. Columns with similar letters are not significantly different.

From the above observations, it may be concluded that shoot tips excised from mature tree responded very well to micropropagation, thus proving to be a potential biotechnological tool for mass production of true-to-type plants of Citrus indica throughout the year without depending on fruiting season and availability of seeds. While considering all the treatments and parameters studied it was found that for both shoot and root initiation combination effect of auxin + cytokinins did not shows significant advantage. On the contrary singular use of cytokinin for shoot initiation and auxins for root initiation were more effective. Shoot tips from mature trees of Citrus indica inoculated on MS media fortified with BAP $0.5 \mathrm{mg} /$ and IBA $1 \mathrm{mg} /$ cultured at $25 \pm 2{ }^{\circ} \mathrm{C}$, with $60 \pm 5$ percent relative humidity with photoperiod of 16 hours proved to be the most effective treatments for shoot induction and root induction respectively. 


\section{Acknowledgement}

The authors are grateful to the Department of Biotechnology, Ministry of Science \& Technology, Govt. of India, New Delhi, India for funding the research project.

\section{References}

Al-Khayri JM and Al-Bahrany AM (2005) In vitro micropropagation of Citrus aurantifolia (lime). Curr. Sci. 81: 1242-1246.

Atiyeh RM, Lee S, Edwards CA, Arancon NQ and Metzger JD (2002) The influence of humic acids derived from earthworm-processed organic wastes on plant growth. Bioreresour Technol. 84: 7-14

Eng WH, Aziz MA and Sinniah UR (2014) Shoot regeneration of Limau purut (Citrus hystrix) using shoot tip: Assessment of calcium gluconate and Silver nitrate in overcoming premature leaf senescence. Pak. J. Bot. 46(4): 1453-1458.

Goswami K, Sharma R, Singh PK and Singh G (2013) Micropropagation of seedless lemon (Citrus limon L.CV. Kaghzi Kalan) and of genetic fidelity of micropropagated plants using RAPD markers. Physio. Mol. Biol. Plants. 19(1): 137-145.

Kangabam L, Thokchom R, Naoroibam SD and Nigthoujam SD (2020) Plant bioregulators and its effect on growth and development of Kachai lemon (Citrus jambhiri Lush.) in vitro. Pharm. J. 9(11):18-21.

Kaur S (2016) In vitro plant regeneration in rough lemon (Citrus jambhiri Lush.) through epicotyls segments by direct shoot organogenesis. J. Nat. Appl. Sci. 8(2): 724-729.

Landau S and Everitt BS (2003) A Handbook of Statistical Analyses using SPSS, New York: Chapman and Hall/CRC, https://doi.org/10.1201/9780203009765

Lombardo G, Alessandro R, Scialabba A and Sciandra M (2011) Direct organogenesis from cotyledons in cultivars of Citrus clementina Hort. Ex Tan. American Journal of Plant Sciences. 2: 237-244.

Malik SK, Chaudhury R, Dhariwal OP and Kalia RK (2006) Collection and characterisation of Citrus indica Tanaka and C. macroptera Mont.-Wild endangered species of Northeast India. Genet. Resour. Crop Ev. 53: 1485-1493.

Muscolo A, Panuccio MR, Abenavoli MR, Concheri G and Nardi S (1996) Effect of molecular complexity and acidity of earthworm faeces humic faections on glutamate dehydrogenase, glutamine synthetase and phosphophenolpyruvate carboxylase in Daucas carotaa II cell. Biol. Fertile soils. 22:83-88.

Perez-Tornero O, Tallon CI and Porras I (2009) An efficient protocol for micropropagation of lemon (Citrus limon) from mature nodal segments. Plant Cell, Tissue Organ Cult. 100(3): 263271.

Rattanpal HS, Kaur G and Gupta M (2011) In vitro plant regeneration in rough lemon (Citrus jambhiri Lush.) by direct organogenesis., African J. Biotechnol. 10: 13724-13728.

Saini HK, Gill MS and Gill MIS (2010) Direct shoot organogenesis and plant regeneration in rough lemon (Citrus jambhiri Lush.), Indian J. Biotechnol. 9: 419:423. 
Sangma YA, Pereira LS, Dang JC and Mathew B (2020) Evaluation of explants for in vitro propagation of Citrus indica Tanaka-An Endangered species, Plant Tissue Cult. Biotechnol. 30(1): 87-96.

Schinor EH, Azevedo FA, Filho FDAM and Mendes BMJ (2011) In vitro organogenesis in some citrus species. Rev. Bras. Frutic. 33(2): 526-531.

Singh IP and Singh S (2003) Exploration, collection and maping of Citrus genetic diversity in India. Technical Bulletin No. 7, National Research Centre for Citrus, Nagpur. pp.230.

Tallon CI, Porras I and Perez-Tornero O (2009) Effect of different phytohormones on the in vitro propagation and rooting of Citrus macrophylla. II International Symposium on Citrus Biotechnology 892: 295-300.

Upadhyay A, Chaturvedi SS and Tiwari BK (2016) Utilization of wild Citrus by Khasi and Garo tribes of Meghalaya. Indian J. Tradit. Know. 15(1): 121-127.

(Manuscript received on 29/11/2020; revised on 22/05/2021) 